$\left(a_{1}\right)_{r}, \cdots,\left(a_{t-1}\right)_{r}$ (and $\left.\mathfrak{a}\right)$ are determined uniquely up to similarity. Then the Krull-Remak-Schmidt theorem, applied now to $\mathfrak{N}_{0} / \mathfrak{M}$, shows that $\left(a_{t}\right)_{r}$ is also determined uniquely up to similarity.

REMARK. The above uniqueness theorem is unsatisfactory, since two diagonal forms (of the same type) with diagonal elements $a_{1}, a_{2}, \cdots$ and $a_{1}^{\prime}, a_{2}^{\prime}, \cdots$ are in general not equivalent (associate) even if $a_{1}, a_{2}, \cdots$ and $a_{1}^{\prime}, a_{2}^{\prime}, \cdots$ are similar in pairs. But if, moreover, $a_{1}$ (therefore also $a_{1}^{\prime}$ ) is a unit, then they are equivalent.*

The Institute for Advanced Study

\title{
ON A MIXED BOUNDARY CONDITION FOR HARMONIC FUNCTIONS
}

HILLEL PORITSKY

In two recent notes in this Bulletin $\nmid$ (referred to below as I, II) I considered the boundary conditions

$$
\frac{\partial u}{\partial n}+a u=0, \quad a=\text { const. }
$$

for harmonic functions, investigating in particular the "reflection" of singularities across a plane at which (1) obtains and indicating several applications of the results.

Dr. A. Weinstein has kindly called my attention to an application of (1) that I have overlooked, namely, to the problem of gravity surface waves of liquids. Under the assumption of small irrotational motion, the velocity potential $\phi$ satisfies along the free boundary the condition $\ddagger$

$$
\frac{\partial^{2} \phi}{\partial t^{2}}+g \frac{\partial \phi}{\partial n}=0 .
$$

For simple harmonic motions with time entering as $e^{i \sigma t}$, this reduces to (1) with

$$
a=-\sigma^{2} / g \cdot \S
$$

Again, equation (1) may be applied, for two-dimensional motions, to the flow function $\psi$ which is the conjugate harmonic to $\phi$ by assuming

* Fitting, loc. cit.

$\dagger$ I, this Bulletin, vol. 43 (1936), p. 873; II, this Bulletin, vol. 44 (1938), p. 443.

$\ddagger$ Lamb, Hydrodynamics, Cambridge, 1924, p. 342.

$\S$ Lamb, loc. cit., p. 342 . 
a wave propagating without change of shape in the direction of $x$ with velocity $c$. Replacing $\partial^{2} / \partial t^{2}$ in (2) by $c^{2} \partial^{2} / \partial x^{2}$, introducing $\psi$, and integrating over the free boundary, one proves that (1) applies to $\psi$ with

$$
a=-g / c^{2}
$$

It will be noted that both in equation (3) and in equation (4) $a$ is negative.

Turning to the "Green's functions" of I, we interpret the one of $\S \S 2-4$ as the velocity potential due to a pulsating point source; the first two functions described in $\$ 5$ would represent the velocity potential due to a small sphere which is oscillating parallel and normally to the free surface; while the two-dimensional Green's function of $\$ 6$ could be used either for a pulsating line source or a line source moving with uniform velocity. In all cases the singularities are at a depth $h$ below the free surface. The interpretation of these functions given in I for the case $R(a)>0$, if it applied now, would replace the effect of the free boundaries by proper images of the sources in an infinite fluid. However, it no longer applies, since the integrals (11), (23), . . no longer converge. On the other hand, the representations such as (18), (23), (24) still apply, provided the path of integration be properly deformed in the complex plane. Similarly, equation (25) still applies.

For a canal of finite depth $H$ the following boundary conditions apply at the bottom $y=0$ :

$$
\begin{aligned}
\psi & =0, \\
\frac{\partial \phi}{\partial n} & =0 .
\end{aligned}
$$

The last boundary condition along with the boundary condition (1) I investigated in II, $\$ 3$, once more as regards the "reflection" of singularities, and again confining myself to the case $R(a)>0$. Dr. Weinstein has considered the boundary conditions of the form (1) and (5) along the two parallel boundaries of a strip and for negative $a{ }^{*} \mathrm{He}$ showed that except possibly for the functions

$$
\sinh \alpha y \sin \alpha x, \sinh \alpha y \cos \alpha x
$$

there exist no bounded non-null solutions of the problem. Obviously (7) will satisfy (1) at $y=H$ provided

* Rendiconti Lincei, 1927, p. 259; Comptes Rendus de l'Académie des Sciences, Paris, 1927, p. 479; Die Naturwissenschaften, 1929, p. 381. 


$$
\alpha \operatorname{coth} \alpha H+a=0 . *
$$

A different treatment of the same subject was given by Hoheisel $\dagger$ who first reflects across the boundary $y=0$ then reflects the function $\partial u / \partial y+a u$ across the other boundary $y=H$ in a manner entirely similar to the one employed in I, $\$ 2$, II, $\S 3$. Hoheisel is led to a differencedifferential equation for the harmonic function involving its values and its $y$ derivatives at $(x, y)$ and $(x, y+2 H)$.

The above is of interest in connection with the results of I, II in pointing out further the difference that might exist between a case where only the real part of $a$ is positive and when it is negative. The existence of harmonic solutions of the proper homogeneous boundary conditions would render the corresponding Green's functions nonunique to within an additive linear combination of these solutions. From Weinstein's results it follows, therefore, that the Green's function of II, $\$ 3$, if existent for $a<0$ and bounded at infinity, is also unique. The solutions (7), (8) of Weinstein are the residues of the integrands of the integrals of the form II, (17).

General Electric Co., ScheneCtady, N. Y.

* The surface waves represented by the above were investigated first by Airy (see Lamb, loc. cit., §§227-229). Equation (5) of 229 takes the place of the above (8) due to the fact that the velocity potential $\phi=\cosh \alpha y \sin \alpha x$ rather than the flux function (7) is employed.

$\dagger$ Jahresbericht der deutschen Mathematiker-Vereinigung, vol. 39 (1930), p. 54. For this reference I am also indebted to Dr. Weinstein. 